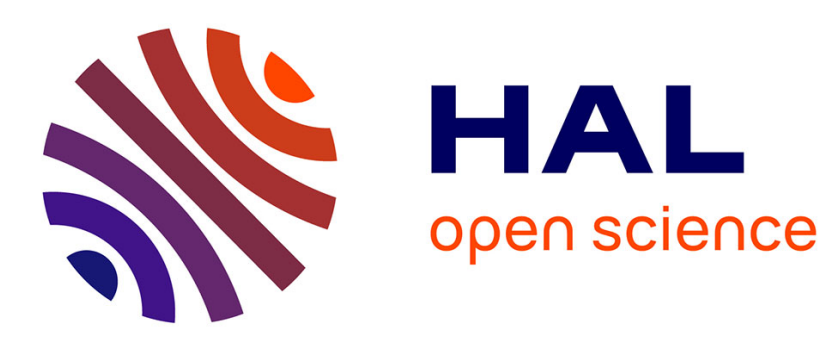

\title{
Adsorption in complex porous networks with geometrical and chemical heterogeneity
}

Quang-Tri Doan, Grégory Lefèvre, Olivier Hurisse, François-Xavier Coudert

\section{To cite this version:}

Quang-Tri Doan, Grégory Lefèvre, Olivier Hurisse, François-Xavier Coudert. Adsorption in complex porous networks with geometrical and chemical heterogeneity. Molecular Simulation, 2014, 40 (1-3), pp.16-24. 10.1080/08927022.2013.844344 . hal-02113365

\section{HAL Id: hal-02113365 https://hal.science/hal-02113365}

Submitted on 28 Apr 2019

HAL is a multi-disciplinary open access archive for the deposit and dissemination of scientific research documents, whether they are published or not. The documents may come from teaching and research institutions in France or abroad, or from public or private research centers.
L'archive ouverte pluridisciplinaire HAL, est destinée au dépôt et à la diffusion de documents scientifiques de niveau recherche, publiés ou non, émanant des établissements d'enseignement et de recherche français ou étrangers, des laboratoires publics ou privés. 


\title{
RESEARCH ARTICLE
}

\section{Adsorption in complex porous networks with geometrical and chemical heterogeneity}

\author{
Quang-Tri Doan, ${ }^{\mathrm{a}}$ Grégory Lefèvre, ${ }^{\mathrm{a}}$ Olivier Hurisse, ${ }^{\mathrm{b}}$ François-Xavier Coudert ${ }^{\mathrm{a} *}$

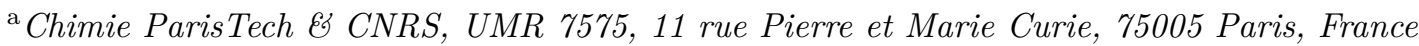 \\ ${ }^{\mathrm{b}}$ EDF RGD, Fluid Dynamics, Power Generation and Environment, 6 quai Watier, 78401 Chatou, France
}

(Received 00 Month 200x; final version received 00 Month 200x)

\begin{abstract}
We report here a simple algorithm to create 2D lattice-based models of porous deposits of preformed nanometric particles, by mimicking to some extent the physics of the actual deposition/aggregation mechanism. The heterogeneous porous networks obtained exhibit anisotropic properties unlike lattice-based models of porous materials in the existing literature, such as those of porous Vycor glass. We have then used calculations based on the mean field kinetic theory, in order to study the thermodynamics and dynamics of fluid adsorption and desorption in these lattice based porous models. We showcase the influence of pore heterogeneity on the phase equilibrium of the confined fluid, studying both heterogeneity in pore size distribution and chemical heterogeneity of the internal surface.
\end{abstract}

Keywords: adsorption; thermodynamics; lattice model; nanoporous materials

\section{Introduction}

When a molecular fluid is confined to spaces of nanoscopic scale, a generic confinement effect is expected to take place in addition to the standard interface effect that arises from the interaction of the fluid molecules with the confined walls. Both effects may lead to drastic changes in the structure, dynamics and thermodynamics of the confined fluid, compared to the bulk fluid. In particular, the understand of confinement of fluids in microporous (diameter smaller than $2 \mathrm{~nm}$ ) and mesoporous (2 to $50 \mathrm{~nm}$ ) materials[1] is a very active field of research, and has lead to the development of a large number of numerical simulation methods with various levels of description of the material, the adsorbates and their interactions.

At the lower side of the scale, atomistic molecular simulation methods have been widely used to characterize adsorption in microporous materials, including the structure of the adsorbed phase, the microscopic details of sorbent/sorbate interactions, dynamics of the adsorbed fluid and thermodynamics of the adsorption. [2,3] As time passed, increase in the computational power available has allowed researchers to extend this method to larger length scales, and provide atomistically detailed descriptions of fluid adsorption in mesoporous materials. [4] On the other end of the complexity scale, the family of methods based on the density functional theory (DFT) have seen a large development in the field of adsorption science since the seminal work of Seaton et al.[5] in 1989. DFT (sometimes called classical DFT, in order to separate it from the quantum chemistry DFT method which focusses on the electron density) describes the density of the adsorbed fluid inside

*Corresponding author. Email: fx.coudert@chimie-paristech.fr 
mesoscopic pores of ideal geometry, allowing to shed light into the thermodynamics of adsorption in a large variety of systems as well as helping interpret experimental adsorption-desorption isotherms.[6]

At an intermediate scale between molecular simulation and DFT methods, lattice-based meanfield models have seen significant interest for the understanding of adsorption and the properties of confined fluid.[7] Such approaches allow to describe non-ideal pore geometries of mesoscopic scale, without the need for an atomistic description of the porous material and adsorbate. They can be used to describe not only the thermodynamics of adsorption near the equilibrium, but also allow insight into the near-equilibrium dynamics of the systems studied using mean field kinetic theory. [8] These methods, spearheaded in the domain of adsorption by the group of Peter Monson, have been successfully used to better understand the nature of adsorption/desorption hystereses for fluids in various mesoporous materials of typical geometries (single pores, "inkbottle pore", "duct pore", closed pores, etc.).[7]

The work presented in this paper has arisen from a practical issue of fundamental importance: provide a better understanding of water ebullition in and near porous solids of complex geometries. In heat exchangers and steam generators, particles settle and form porous deposits. The deposition process of particles depends on multiple factors including $\mathrm{pH}$ and surface roughness,[9-11] and relatively little information is available on the exact geometrical, topological and chemical characteristics of these porous deposits. Understanding the effects of the deposits on evaporation and boiling of water represents an important issue for steam generator operators because such deposits causes a severe reduction in efficiency particularly in steam generators of power plants.[12] Moreover, a good comprehension of the thermodynamics and dynamics of these phenomena is key to include them in a higher-level modeling of the system, for example to account for them in multiphasic computational fluid dynamics.[13]

Our team's earlier approach to this issue was experimental, and we mimicked the porous deposits using a model system made of mono-disperse silica particles and study evaporation and boiling of water confined in the pores of micro-spheres colloidal silica.[14] In the present theoretical work, we combine a new method to randomly generate porous structures by deposition of preformed nanometric particles, and use lattice-based models to study the thermodynamics and kinetics of liquid-gas transitions in these porous spaces of high geometrical and chemical heterogeneity.

\section{Formation of the porous deposits}

\subsection{Generating porous microstructures: an overview}

In the first part of this paper, we describe the method we have developed to generate lattice-based models of the porous space created by deposition of nanoscale particles at a surface immersed in liquid. There are several existing computational and experimental techniques that have been described in the literature to produce model representations of porous materials. Perhaps the most direct methods are based on experimental 2D images, using microstructure reconstruction algorithms in order to obtain plausible 3D structures from a series of 2D micrographs.[15] These methods have been successfully used in disordered mesoporous materials with high geometric heterogeneity, and in particular the very complex structure of porous Vycor glass.[16] Recent developments of direct 3D imaging techniques, including X-ray microtomography or X-ray microscopy, have enabled a more direct access to the microstructure and pore network geometry and topology of mesoporous media,[17] and even at the flow of fluid inside porous media.[18]

In the absence of direct experimental images, it is also possible to generate plausible microscopic structures of porous media by manipulation of random fields, such as controlled smoothing of random white-noise images. [19] By playing with the parameters of smoothing and the number of iterations of the procedure, it is possible to obtain structures of controlled regularity and porosity.

Finally, a third class of methods for the description are those that mimic the real physical process of formation of the actual sample, either in a atomistically-detailed manner or in a more 
abstract way. For example, Gelb and Gubbins[20] proposed to use quench molecular dynamics procedure to mimic the processes by which Vycor glass and controlled-pore glasses are produced. A similar technique was used more recently for porous carbons.[21] Monette et al.[22] had in an earlier work proposed a lattice-based representation using a three-dimensional Ising model with long range interactions.

However, the methods described above have been little applied to the problem of aggregation and deposition of nanoscopic particles of matter to form mesoporous structures. The closest cases treated in the literature concern aerogels and other related high porous aggregates, $[23,24]$ and rely mostly on random disposition of spherical particles. A recent study into the complex microstructure of cement[25] has shown the importance of describing the geometrical heterogeneity of the material (in the case of cement, the polydispersity of the calcium-silicate-hydrate aggregates) to correctly reproduce the known macroscopic properties of the material. In the following sections, we describe a series of procedures we propose to generate two-dimensional lattice-based models of porous aggregates of nanoscopic particles.

\section{$2.2 \quad$ Initial model of deposition}

The first and simplest model we propose to create porous networks by random deposition features heterogeneous "rough" particles of fixed size and random shape, deposited iteratively at random on a planar surface. This model is schematized on Figure 1, and consists of the two following steps:

(1) Generation of a library of particle shapes (upper panel of Fig. 1) - We start by choosing a maximum particle size, $\ell$, and creating a randomly occupied $\ell \times \ell$ lattice (each lattice site is occupied with probability 0.5). Then, a compact particle is created from this random lattice by regrouping the occupied sites in two series of motions: first horizontally (in a random direction), then vertically (again in a random direction). We repeat this process many times in order to obtain a large library of randomly-shaped particles. Their diameter varies, but is guaranteed to be smaller than $\ell$. Moreover, given the initial conditions of the generating algorithm (random occupancy of the lattice), the mean particle size is $\ell / \sqrt{2 \pi} \approx 0.4 \ell$.

(2) Random deposition of particles (lower panel of Fig. 1) - The second step of our model mimics the deposition of previously formed particles onto a planar surface. We start with an empty 2D lattice of dimension $L \times H$, of which the lowest line is considered filled (the planar surface). Then, particles are chosen at random from the previously created library, and dropped one at a time onto the surface at a random position. The first particle drops until it makes contact with the bottom of the lattice. Further particles will then be dropped in a similar fashion, except that they may stop their fall at the first contact with an earlierdeposited particle, or with the planar surface. They will then be frozen in place, much like the irregular falling blocks in the popular Tetris computer game. This process results in an irregular porous space between the particles, and is somewhat similar (although very simplified) to the actual physical process of particle deposition and aggregation.

To our knowledge, it is the first time that such a mechanism for building complex and heterogeneous porous networks is proposed in the literature. Similar attempts to use Tetris-like lattice movements found in the literature dealt with stacking of regularly shaped particles, and focused not on the process of aggregation and formation of porous space, but rather on the compaction of granular media[26] and its resistance to shear.[27]

Figure 2 provides a quick look at four different realizations of the random deposition algorithm described above, with particle size $\ell=10$ and lattice size $50 \times 50$. Clear variations and some common traits can been seen from this figure. First, the porosity of the resulting lattice varies among realizations, going from 0.4 to 0.7 depending on the successive random choices of position for the particles dropped, i.e. a wide distribution of porosity but overall high porosities (or low 
densities). Moreover, the porous system is clearly heterogeneous, with a combination of smaller and larger pockets (enclosed porous voids in our 2D representation) and pores accessible from the outside. However, even with this heterogeneity, some characteristic features of the methodology are shared: the porous system is highly anisotropic, featuring a variable number of vertical "chimneys" due to the deposition process; the porous deposits exhibit an overall vertical gradient in density, being denser near the deposition surface and more porous at their extremity. All these features are in good agreement with the very few direct images available in the literature, such as those from porous corrosion deposits in Seabrook Pressurized Water Reactor. [28] These features are in sharp contrast with the already published methods of generating lattice models of porous solids, which are all isotropic.

\section{$2.3 \quad$ Refinements of the model}

The densities typically obtained from the procedure described in the previous section are quite low compared to what direct images suggest, even though no direct local measurement of density (or porosity) of the real deposits is available, to our knowledge. We thus derived ways to obtain a higher density (lower porosity) by amending the algorithm for the generation of the porous structure. We used three different modifications:

(1) First, multiple iterations of the generating algorithms are run, and all those whose density does not exceed a certain preset threshold are rejected.

(2) The smaller inaccessible pockets of the porous system (up to $3 \times 3$ ) are removed, i.e. the corresponding lattice sites are considered solid. This mimics the aging of the porous deposits, in which any very sharp feature or small pore would disappear after some time due to local dissolution-recrystallization.

(3) Instead of using particles of initial size $\ell$, we create a library of particles of size varying between $\ell=6$ and 12. This increases the polydispersity, and thus the density of the aggregate.[25]

(4) Finally, we amended the Tetris-like random deposition model by adding a condition on the size of the particle-deposit contact area, as a very simple way to account for possible particle resuspension in the fluid. With this condition, we consider that particles deposited onto the already formed deposit but with only a small area of contact with it would lack sufficient adhesion forces to stick, and be resuspended into the solution. Thus, we added the following condition: after a new particle is dropped onto the deposit, if its number of contacts with the existing surface (in units of lattice edges) is lower than 3 , the particle is removed before the next one is added.

By combining these four modifications of the algorithm, we were able to grow denser heterogeneous porous networks, with porosities down to 0.2 (density of 0.8 ), while keeping the characteristic features we wanted in the model: anisotropy and existence of density gradient. We have then performed simulations on the lattice models obtained, as described in the following sections.

\section{Adsorption on these lattice models}

In order to study the behavior of fluid inside our heterogeneous porous space, the effects of the confinement on the fluid's thermodynamics and the dynamics of its phase transition, we used a lattice-based model. In order to keep computational costs reasonable on the large systems that we are interested in, we decided on a mean field approach and followed the mean field kinetic theory (MFKT) equations as applied to a lattice gas model under confinement, using the formalism set forth by Monson in 2008[8] (and similar to the presentation of Gouyet et al.[29]). Later work by Monson and other groups have shown that it is widely applicable to adsorption and desorption in model geometries, $[30,31]$ and has been used to help understand the wetting and drying phenomena 
in porous media.[32, 33]

\subsection{The method: mean field kinetic theory}

The basis for the mean field kinetic theory is a 2D lattice gas model: in such a model, each site of a 2D square lattice is either filled with solid, liquid or vapor. In the nearest neighbor approximation, a Hamiltonian can be constructed by summing up the energy over pairs of neighbors, by neglecting many body effects (which could be incorporated in a more refined Hamiltonian). In this model, the solid is immovable and acts as both an inaccessible volume (that the fluid cannot enter) and an external field on neighboring sites. The simplest Hamiltonian that can be constructed assigns an energy $-\epsilon_{\mathrm{ff}}$ for two neighboring sites occupied by the liquid, and an energy $-\epsilon_{\mathrm{sf}}$ for neighboring liquid and solid sites. Gas-gas, gas-liquid and gas-solid interactions are taken as zero, and Hamiltonian can be written as:

$$
H=-\frac{1}{2} \epsilon_{\mathrm{ff}} \sum_{i} \sum_{a} n_{i} n_{a}-\epsilon_{\mathrm{sf}} \sum_{i} n_{i} \phi_{i}
$$

where $i$ runs over all non-solid sites of the lattice, $a$ runs over the nearest neighbors of $i$ (Figure S1), $n_{i}$ indicates the fluid occupancy of a site ( 0 for gas, 1 for liquid), and $\phi_{i}$ denotes the number of neighboring sites occupied by a solid.

In the mean field approximation, we take as key variable not the occupancy of each site $n_{i}$, but the mean fluid density $\rho_{i}$. The Helmholtz energy for the system is then given by:

$$
F=k T \sum_{i}\left[\rho_{i} \ln \rho_{i}+\left(1-\rho_{i}\right) \ln \left(1-\rho_{i}\right)\right]-\frac{1}{2} \epsilon_{\mathrm{ff}} \sum_{i} \sum_{a} \rho_{i} \rho_{a}-\epsilon_{\mathrm{sf}} \sum_{i} \rho_{i} \phi_{i}
$$

The distribution of fluid at the equilibrium with a given chemical potential $\mu$ is then given by the following self-consistent equation:[8]

$$
\frac{1}{\rho_{i}}=1+\exp \left[-\frac{\mu-\epsilon_{\mathrm{ff}} \sum_{a} \rho_{a}-\epsilon_{\mathrm{sf}} \phi_{i}}{k T}\right]
$$

Resolution of this equation by iterating from a given set of initial conditions yields the steady state of the system, i.e. its density in the limit of infinite time. This steady state is not necessarily the thermodynamic equilibrium of the system, and may correspond (depending on the initial conditions) on a metastable state. We will use these "static" calculations to obtain adsorption and desorption isotherms of fluid in the porous space.

In addition to the static calculations, Monson[8] showed that the dynamics of the system out of the steady state could be described by the mean field kinetic equations. In these equations, the evolution of density in each site is linked to the flow of particles between neighboring sites $J_{i a}$ by:

$$
\frac{\mathrm{d} \rho_{i}}{\mathrm{~d} t}=-\sum_{a} J_{i a}
$$

Considering a Kawasaki dynamics with Metropolis transition probabilities in the mean field approximation, Monson demonstrated that the flux can be written as:

$$
J_{i a}=w_{0} \exp \left(-E_{i a} / k T\right) \rho_{i}\left(1-\rho_{j}\right)-w_{0} \exp \left(-E_{a i} / k T\right) \rho_{j}\left(1-\rho_{i}\right)
$$

where $E_{i a}=\max \left[0, E_{a}-E_{i}\right]$, and $w_{0}$ is the jump rate between neighboring sites in the absence of interactions. It sets the time scale of the dynamics.

In the following sections, we will use static and dynamic MFKT calculations to study the influence of confinement in complex heterogeneous pores on liquid-gas phase transitions. 


\subsection{Results in complex porous network: static calculations}

We start by characterizing adsorption and desorption in a porous deposit constructed by the algorithm presented above, on a $100 \times 100$ lattice with overall porosity of 0.3 (density of 0.7 ). We illustrate this behavior in the case of a hydrophilic solid, i.e. one for which the fluid-wall interaction is more important than fluid-fluid interactions; we took values of $\epsilon_{\mathrm{ff}}=k T$ and $\epsilon_{\mathrm{sf}}=3 \epsilon_{\mathrm{ff}}$. Static calculations for adsorption (respectively desorption) isotherms were performed by successive small increments (respectively decrements) of the activity $\lambda=\exp (\mu / k T)$, which we assimilate to the fluid pressure $(\lambda \simeq P)$ in the following for simplicity of the discussion.

The upper panel of Figure 3 presents the adsorption and desorption isotherms, while the lower panel is composed of snapshots of the system at specific points in the isotherms (see figure caption for details). Two regions are clearly present in the isotherms: a reversible uptake up to $P / P^{0}=0.5$, then a marked step in the isotherms coupled with a wide hysteresis loop. This behavior is markedly different from that of adsorption-desorption in a slit pore of similar average dimension $H$ and finite length $L$ (whose isotherms are depicted in Figure S2, for $H=6$ and $L=20$ ). In the case of the slit pore of relatively small size, as detailed for example in ref. [8], there are two steps: the first associated with the formation of a monolayer of fluid on the walls, the second associated with the metastability of liquid and vapor states in the pore. Both features are clearly different between the model slit pore and our complex porous system. The initial part of the isotherm in the latter case does not feature a step, but rather a Langmuir-type uptake at $P / P^{0}<0.5$ : this corresponds to the filling of the smaller pores of the system, some of which are of much lower size than the average pore size. Thus, the pore size heterogeneity and the presence of some very small pores account for the large attraction of the fluid by the porous deposit at low pressure. At higher pressure, the hysteresis loop between adsorption and desorption branches is both quantitatively and qualitatively different from that of the slit pore. First, because of the pore size heterogeneity, it is wider. Secondly, instead of two almost-vertical transitions, we observe a quite smooth adsorption branch and a steeper (but not vertical) desorption branch. Again, this stems from the large pore size distribution, which leads to rounding because adsorption in different pores happens at different pressure. Moreover, the disymmetry between adsorption and desorption branches clearly reflects the different nature of pore filling and emptying, as seen in the snapshots (lower panel of Fig. 3) in the middle of the hysteresis. The desorption branch is steeper than the adsorption branch, because the mechanism for pore emptying is not the same as pore filling: during pore filling, the adsorbed film on the walls grow until both sides join; upon pore emptying, an interface (meniscus) is formed which then quickly recedes, leading to a sharper transition.

\subsection{Results in complex porous network: dynamics}

In order to better understand the effect of porous network heterogeneity on the liquid-vapor transition, we performed dynamic MFKT calculations. We highlight here one specific example of such, in which a porous deposit of low density featuring long vertical chimney-like pores (see Figure 4), initially filled with vapor, is surrounded by liquid at time $t=0$. We report on Fig. 4 the time evolution of the density inside the pores, as well as snapshots of the system at different times. First, we see that not all the pores of our 2D model are filled: the porous space includes some closed "pockets", inaccessible from the outside. While, in a static equilibrium calculation, these are filled, the fluid obviously cannot flow into them during our dynamics. This effect, which accounts for the low density $(\rho=0.61)$ reached at $t \rightarrow \infty$, is particularly visible because our model is two-dimensional and the connectivity of each site is only four. Apart from that, we see that the dynamics of pore filling is actually pretty homogeneous, with two different time scales: the initial filling of the outer regions of the porosity (up to $\rho \simeq 0.3$ ) happens very rapidly (simulation time of $w t \leq 200$ ), while the gradual filling of the accessible interior pores happens gradually and more slowly, with the longer pores being the longer to be fully filled. Overall, the dynamics of pore filling is pretty featureless, and much more gradual that the steeped uptake dynamics typically reported for simple pore geometries. $[7,8]$ 


\subsection{Chemically heterogeneous pores}

We focus in this section on porous materials whose heterogeneity comes not only from their topology, but also from the chemical nature of their accessible surface. First, we showcase the method on a simple model of mixed hydrophobic-hydrophilic material: a 2D slit pore with checkered surface, i.e. series of alternating hydrophobic and hydrophilic domains of fixed size (see Figure 5 for illustration). We performed static calculations of adsorption and desorption isotherms on systems with increasing domain size, with wall-fluid interactions equal to $\epsilon_{\mathrm{sf}}=3 \epsilon_{\mathrm{ff}}$ for the hydrophilic domains and $\epsilon_{\mathrm{sf}}=0$ for the hydrophobic domains, and a slit pore height of $H=6$. For domain sizes larger than or equal to 5, we observe a three-step adsorption (and corresponding three-step desorption), with: (i) formation of a layer atop the hydrophilic domains; (ii) bridging of the opposite domains to form a fluid film; (iii) junction of the neighboring bridges to fill the whole pore, with lower fluid density directly atop the hydrophobic domains (see Figure 5). On the other hand, for small domain sizes (compared to slit pore height), this effect is not seen and the checkered surface behave as a globally "neutral" with respect to water, i.e. neither hydrophilic nor hydrophobic: after adsorption of the first monolayer on top of hydrophilic domains (local effect), adsorption happens in one steep transition corresponding to homogeneous filling of the pore. The adsorption-desorption isotherms display a broad hysteresis around $P=P^{0}$. These findings confirm the suitability of the method used to treat such questions and exemplify the diversity of behaviors that can arise from chemical heterogeneity in pore surfaces, even in the case of the relatively simple slit pore.

Finally, we wanted to see the effect of a combination of geometrical and chemical heterogeneity in a truly complex porous deposit. We thus amended the Tetris-like deposition algorithm in order to produce some deposits that would include both hydrophobic and hydrophilic nanoparticles (in an equimolar mixture). We retained the identity of each of the aggregated particles in the final porous network, yielding a mixed hydrophobic-hydrophilic porous network with complex geometry (depicted in Figure 6). Our initial results on this system include a series of static adsorption and desorption calculations, whose isotherms are plotted in Fig. 6 along with some snapshots of the system at various points during adsorption (top) and desorption (bottom). First, we see that low-pressure adsorption is unaffected by the heterogeneity: reversible adsorption of a first layer of fluid on the hydrophilic domains. The only difference in that part is that its absolute uptake is scaled down by a factor of nearly two, accounting for the reduced hydrophilic accessible surface area. However, the higher-pressure hysteresis loop in the adsorption-desorption isotherms is pretty different from the purely hydrophilic case (compare Fig. 6 with Fig. 3). Overall, the transition is pushed to higher pressure, near $P=P^{0}$, reflecting an overall neutral character of the pore surfaces. Moreover, both the adsorption and desorption branches are now rather smooth, instead of the steep desorption branch of Fig. 3. This demonstrates that the presence of the hydrophobic domains in the composite deposit has modified the mechanism of the desorption transition. We can see clearly in Fig. 6 that the random occurrence of neighboring hydrophilic domains leads to the persistence of liquid films bridging them upon desorption, something that does not occur in the pore system with homogeneous surface. Further work now needs to focus on characterizing the pore filling and pore emptying dynamics of this complex system, in order to see whether this equilibrium behavior persists.

\section{Conclusions}

In conclusion, we have constructed a simple algorithm to create $2 \mathrm{D}$ lattice-based models of porous deposits of preformed nanometric particles, by mimicking to some extent the physics of the actual deposition/aggregation mechanism. The heterogeneous porous networks obtained exhibit anisotropic properties unlike lattice-based models of porous materials demonstrated in the literature, such as those of porous Vycor glass. We have then used calculations based on the mean field kinetic theory, in order to study the thermodynamics and dynamics of fluid adsorption and desorption in these lattice based porous models. We showcase the drastic influence of pore het- 
erogeneity on the phase equilibrium of the confined fluid, with a focus on two different kinds of heterogeneity: the complex pore geometry and topology, and the chemical heterogeneity (hydrophobicity and hydrophilicity) of the internal surface of the porous deposits. Some of the perspectives opened by this work include some refinements of the model construction algorithm, such as the extension to 3D systems (at the price of much larger computational resources for adsorption studies) and the simulation of an aging (or ripening) of the material, akin to the experimental dissolution-recrystallization phenomenon. Regarding the dynamics of the pore filling and emptying, we currently have seen relatively little influence of the pore heterogeneity on the mean field dynamics: it would be worth researching whether this can lead to larger effects in individual events, for example by actually running many realizations of individual Kawasaki dynamics of the system.

\section{Acknowledgments}

This research was financially supported by EDF R\&D, Department of Fluid Dynamics, Power Generation and Environment, under the umbrella of project MOPHET-C (refined modeling of phenomena related to thermohydraulics and chemistry, MOdélisation Fine des PHÉnomènes en Thermohydraulique-Chimie), whose aim is to improve the understanding of particle fouling, develop physical models and perform numerical simulations of particle deposit formation in turbulent flows, as occurred for instance in steam generators. The authors are grateful to Chantal Hervouet and Jean-Pierre Minier for discussions about the physical and chemical characteristics of steam generator deposits, and the relevance of the theoretical models and methods developed to real systems. FXC thanks Rodolphe Vuilleumier for fruitful discussions on the effect of confinement on phase transitions.

\section{References}

[1] A.D. McNaught and A. Wilkinson, IUPAC. Compendium of Chemical Terminology, 2nd ed. (the "Gold Book"). Wiley Blackwell; 2nd Revised edition edition, 1997.

[2] C.M. Lastoskie and K.E. Gubbins, Characterization of porous materials using molecular theory and simulation, Advances in Chemical Engineering 28 (2001), pp. 203-250.

[3] C.R.A. Catlow, B. Smit, and R.A. van Santen, Computer Modelling of Microporous Materials Elsevier Ltd., 2004.

[4] P.A. Monson, Recent Progress in Molecular Modeling of Adsorption and Hysteresis in Mesoporous Materials, Adsorption 11 (2005), pp. 29-35.

[5] N. Seaton, J. Walton, and N. Quirke, A new analysis method for the determination of the pore size distribution of porous carbons from nitrogen adsorption measurements, Carbon 27 (1989), pp. 853-861.

[6] J. Landers, G.Y. Gor, and A.V. Neimark, Density functional theory methods for characterization of porous materials, Colloids and Surfaces A: Physicochemical and Engineering Aspects (2013) in press, DOI: 10.1016/j.colsurfa.2013.01.007.

[7] P. Monson, Understanding adsorption/desorption hysteresis for fluids in mesoporous materials using simple molecular models and classical density functional theory, Microporous and Mesoporous Materials 160 (2012), pp. 47-66.

[8] P.A. Monson, Mean field kinetic theory for a lattice gas model of fluids confined in porous materials, J. Chem. Phys. 128 (2008), p. 084701

[9] L. Cerovic, G. Lefèvre, A. Jaubertie, M. Fedoroff, and S. Milomjic, Deposition of hematite particles on polypropylene walls in dynamic conditions, J. Colloid Interface Sci. 330 (2009), p. 284.

[10] C. Henry, J.P. Minier, G. Lefèvre, and O. Hurisse, Numerical Study on the Deposition Rate of Hematite Particle on Polypropylene Walls: Role of Surface Roughness, Langmuir 27 (2011), p. 4603.

[11] C. Henry, J.P. Minier, and G. Lefèvre, Towards a description of particulate fouling: From single particle deposition to clogging, Advances in colloid and interface science 185-186 (2012), pp. 34-76.

[12] S.J. Green and G. Hetsroni, PWR steam generators, Int. J. Multiphase Flow 21 (1995), p. 1.

[13] J.M. Hérard and O. Hurisse, A fractional step method to compute a class of compressible gas-liquid flows, Computers \& Fluids 55 (2012), pp. 57-69.

[14] J. Peixinho, G. Lefèvre, F.-X. Coudert and Olivier Hurisse, "Water evaporation in silica colloidal deposits", submitted to J. Colloid Interface Sci.

[15] D.P. Bentz and N.S. Martys, Hydraulic Radius and Transport in Reconstructed Model Three-Dimensional Porous Media, Transport in Porous Media 17 (1995), pp. 221-238.

[16] D.P. Bentz, E.H. Garboczy, and D.A. Quenard, Modelling drying shrinkage in reconstructed porous materials: application to porous Vycor glass, Modelling Simul. Mater. Sci. Eng. 6 (1998), pp. 211-236.

[17] P. Levitz, V. Tariel, M. Stampanoni, and E. Gallucci, Topology of evolving pore networks, Eur. Phys. J. Appl. Phys. 60 (2012), p. 24202.

[18] S. Berg, H. Ott, S.A. Klapp, A. Schwing, R. Neiteler, N. Brussee, A. Makurat, L. Leu, F. Enzmann, J.O. Schwarz, M. 
Kersten, S. Irvine, and M. Stampanoni, Real-time 3D imaging of Haines jumps in porous media flow, Proceedings of the National Academy of Sciences 110 (2013), pp. 3755-3759.

[19] P.A. Crossley, L.M. Schwartz, and J.R. Banavar, Image-based models of porous media: Application to Vycor glass and carbonate rocks, Appl. Phys. Lett. 59 (1991), p. 3553.

[20] L.D. Gelb and K.E. Gubbins, Characterization of Porous Glasses: Simulation Models, Adsorption Isotherms, and the Brunauer-Emmett-Teller Analysis Method, Langmuir 14 (1998), pp. 2097-2111.

[21] Y. Shi, A mimetic porous carbon model by quench molecular dynamics simulation, J. Chem. Phys. 128 (2008), p. 234707 .

[22] L. Monette, G. Grest, and M. Anderson, Three-dimensional Ising system with long-range interactions: A computer model of Vycor glass, Phys. Rev. E 50 (1994), pp. 3361-3369.

[23] I. Varga and F. Kun, Computer method for modeling the microstructure of aerogel, CMM-2011, Computer Methods in Mechanics (2011).

[24] A. Kharaghani, C. Kirsch, T. Metzger, and E. Tsotsas, Micro-scale fluid model for drying of highly porous particle aggregates, Computers \& Chemical Engineering 52 (2013), pp. 46-54.

[25] E. Masoero, E. Del Gado, R.J.M. Pellenq, F.J. Ulm, and S. Yip, Nanostructure and Nanomechanics of Cement: Polydisperse Colloidal Packing, Phys. Rev. Lett. 109 (2012).

[26] E. Caglioti, V. Loreto, H. Herrmann, and M. Nicodemi, A "Tetris-Like" Model for the Compaction of Dry Granular Media, Phys. Rev. Lett. 79 (1997), pp. 1575-1578.

[27] M. Piccioni, V. Loreto, and S. Roux, Criticality of the "critical state" of granular media: Dilatancy angle in the Tetris model, Phys. Rev. E 61 (2000), pp. 2813-2817.

[28] J. Deshon, PWR Axial Offset Anomaly (AOA) Guidelines, Revision 1, EPRI Technical Report \#1008102, p. 51 (2004).

[29] J.F. Gouyet, M. Plapp, W. Dieterich, and P. Maass, Description of far-from-equilibrium processes by mean-field lattice gas models, Advances in Physics 52 (2003), pp. 523-638.

[30] P.A. Monson, Contact Angles, Pore Condensation, and Hysteresis: Insights from a Simple Molecular Model, Langmuir 24 (2008), pp. 12295-12302.

[31] J. Edison and P. Monson, Dynamic mean field theory of condensation and evaporation in model pore networks with variations in pore size, Microporous and Mesoporous Materials 154 (2012), pp. 7-15.

[32] J.R. Edison and P.A. Monson, Modeling Relaxation Processes for Fluids in Porous Materials Using Dynamic Mean Field Theory: An Application to Partial Wetting, J Low Temp Phys 157 (2009), pp. 395-409.

[33] J.R. Edison and P.A. Monson, Dynamic mean field theory of condensation and evaporation processes for fluids in porous materials: Application to partial drying and drying, Faraday Discuss. 146 (2010), p. 167. 
Figures

1. Particles formation

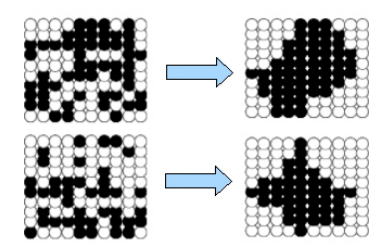

2. Particles deposition

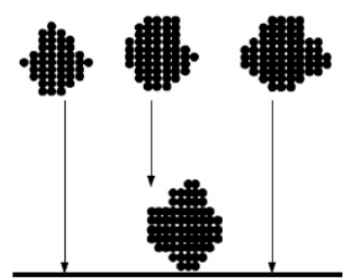

Figure 1. Scheme of a two-step method to generate porous networks by random deposition features heterogeneous "rough" particles.

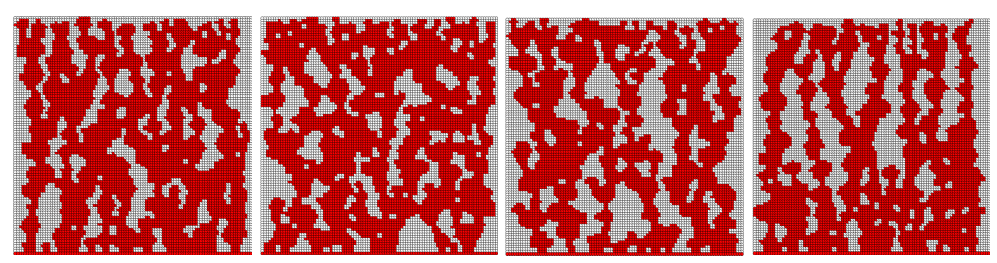

Figure 2. Four realizations of the porous generation method, with particle size $\ell=10$ and lattice size $50 \times 50$. 


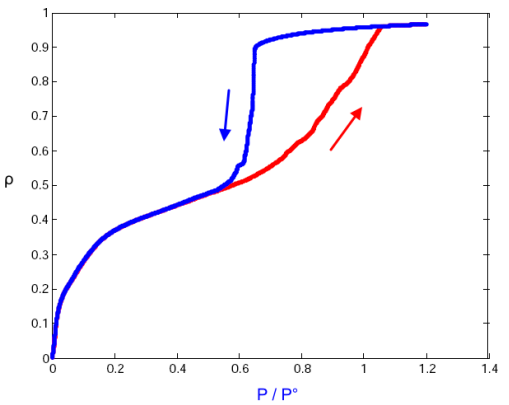

adsorption
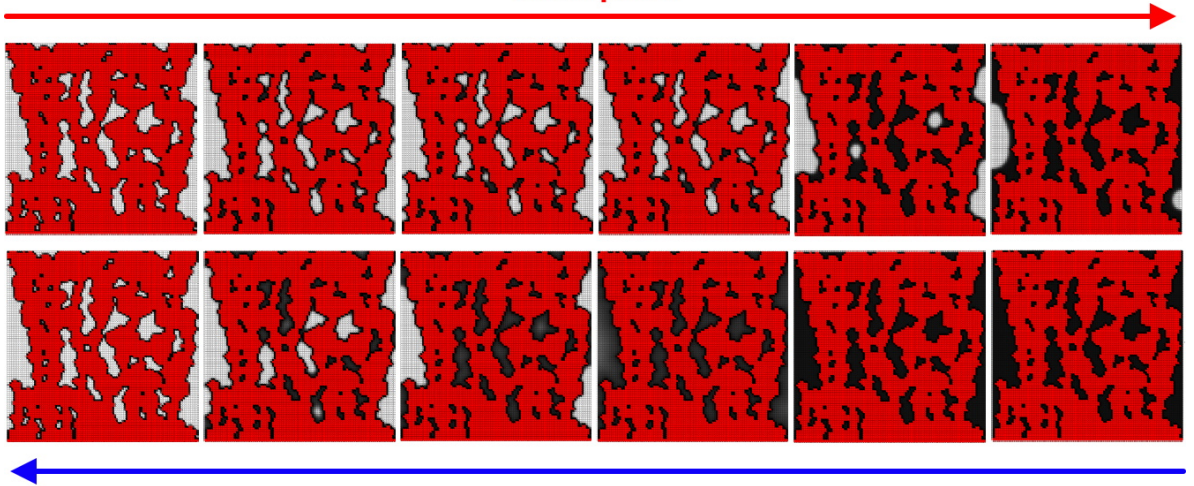

desorption

Figure 3. Upper panel: adsorption and desorption isotherms from static calculations on a disordered pore system. Lower panel: snapshots of the system at relative pressures of $P / P^{0}=0.2,0.64,0.65,0.9$ and 1 ; red $=$ solid, grayscale indicates the fluid density (light gray $=$ vapor, black $=$ liquid)
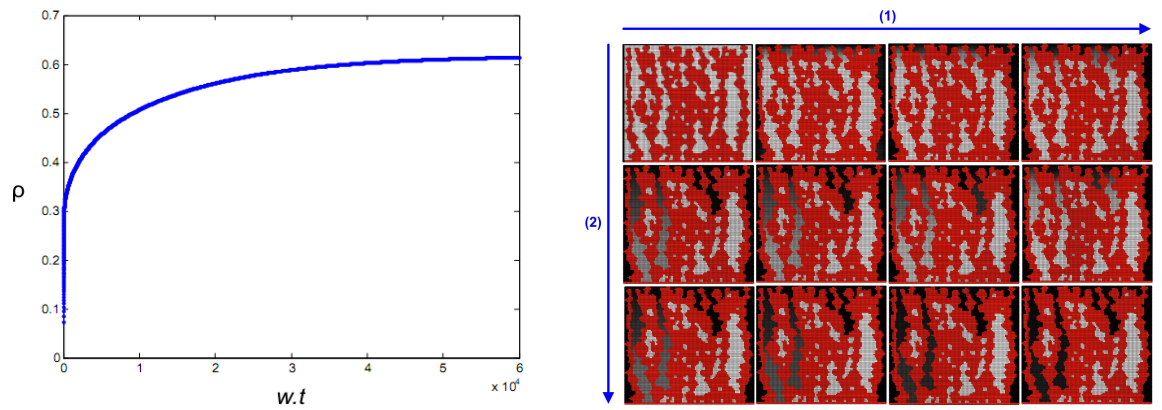

Figure 4. Left: fluid uptake during dynamics of pore filling, as a function of simulation time $w t$. Right: snapshots of the porous system during pore filling (left to right, top to bottom). 

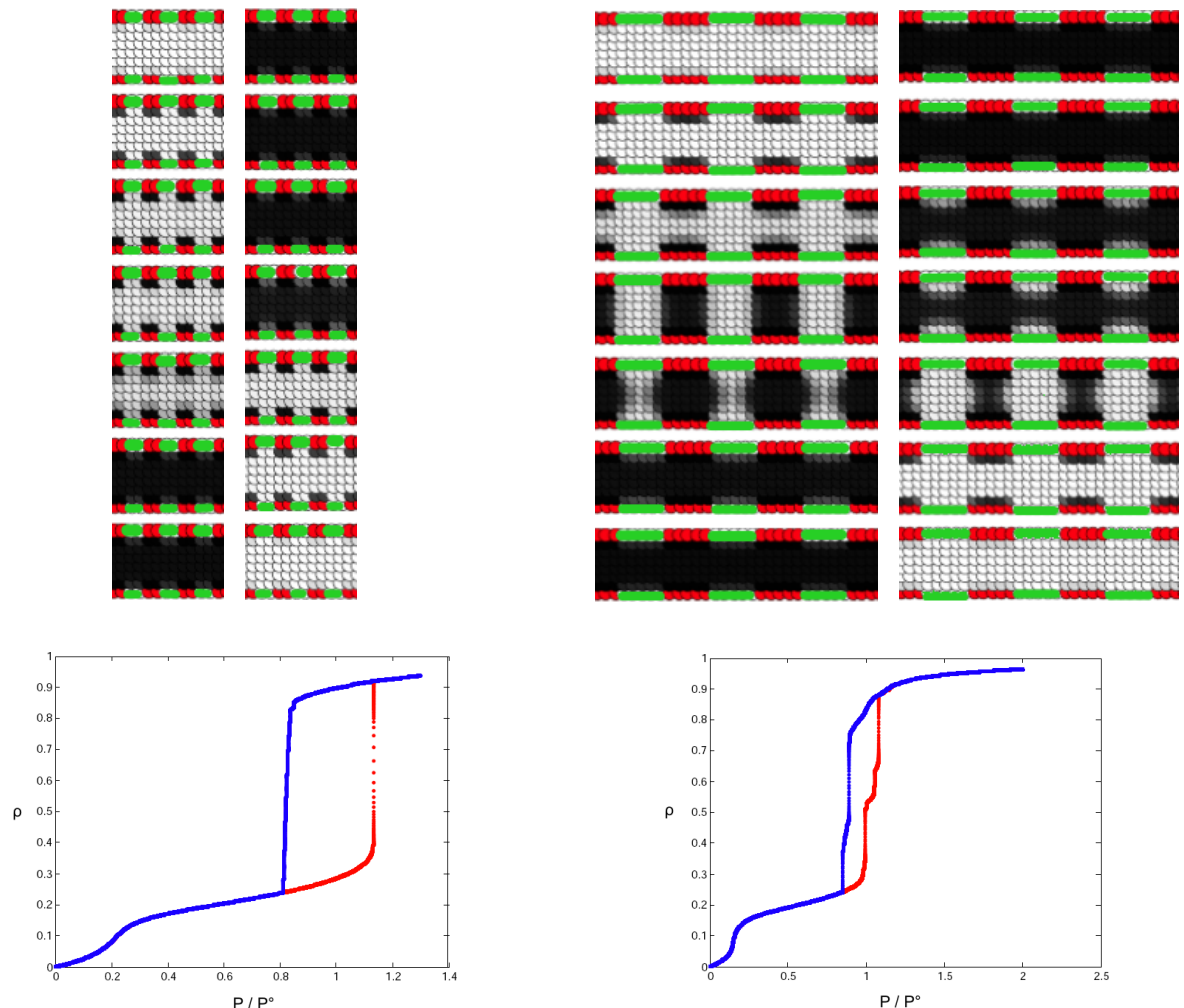

Figure 5. Adsorption and desorption isotherms, and corresponding snapshots, on a checkered hydrophobic-hydrophilic slit pore (see text for details). Left: each domain has length 2 ; right: each domain has length 5 . Key for snapshot colors: red $=$ hydrophilic solid, green $=$ hydrophobic solid, grayscale indicates fluid density (light gray $=$ vapor, black $=$ liquid).
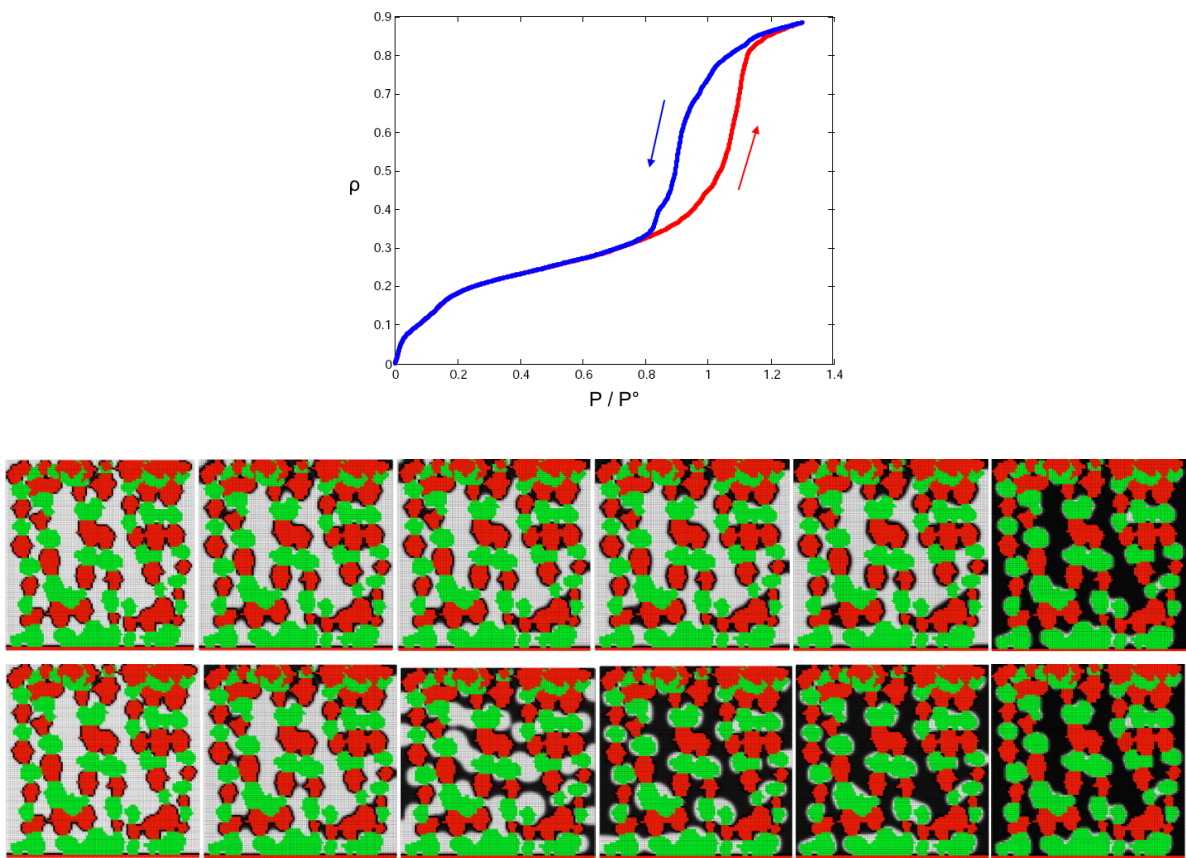

Figure 6. Upper panel: adsorption and desorption isotherms from static calculations on a chemically heterogeneous porous deposit. Lower panel: snapshots of the system during adsorption (top) and desorption (bottom); red = hydrophilic solid, green $=$ hydrophobic solid, grayscale indicates fluid density (light gray $=$ vapor, black $=$ liquid $)$ 